\title{
Development of Algorithms for Automated Timed Up-and-Go Test Subtask and Step Frequency Analysis
}

\author{
Ane DIZ FELIPE ${ }^{\mathrm{a}, \mathrm{b}, \mathrm{c}}$, Andreas ZIEGL ${ }^{\mathrm{a}, \mathrm{b}, 1}$, Dieter HAYN ${ }^{\mathrm{a}, \mathrm{d}}$ and Günter SCHREIER SH $^{\mathrm{a}, \mathrm{b}}$ \\ a AIT Austrian Institute of Technology GmbH, Graz, Austria \\ ${ }^{\mathrm{b}}$ Graz University of Technology, Austria \\ ${ }^{c}$ Universidad Politécnica de Madrid, Spain \\ ${ }^{d}$ Ludwig Boltzmann Institute for Digital Health and Prevention, Salzburg, Austria
}

\begin{abstract}
Frailty is one of the major problems associated with an aging society. Therefore, frailty assessment tools which support early detection and autonomous monitoring of the frailty status are heavily needed. One of the most used tests for functional assessment of the elderly is the "Timed Up-and-Go" test. In previous projects, we have developed an ultrasound-based device that enables performing the test autonomously. This paper described the development and validation of algorithms for detection of subtasks (stand up, walk, turn around, walk, sit down) and for step frequency estimation from the Timed Up-and-Go signals. The algorithms have been tested with an annotated test set recorded in 8 healthy subjects. The mean error for the developed subtask transition detection algorithms was in between 0.22 and $0.35 \mathrm{~s}$. The mean step frequency error was $0.15 \mathrm{~Hz}$. Future steps will include prospective evaluation of the algorithms with elderly people.
\end{abstract}

Keywords. Frailty, timed up-and-go test, tele-health, monitoring

\section{Introduction}

The growth in life expectancy in the past decades has led to increasing frailty, which is a risk factor for many diseases [1]. To prevent the associated negative health outcomes, an early identification of the frailty signs and symptoms is needed. There are various approaches for automatically assessing frailty with different devices, which are very well summarized in [2] and [3]. One of the most promising frailty-assessment tools especially for home-based, autonomous scenarios, is the Timed Up-and-Go (TUG) test [4]. While the total TUG time has already proven to be a good frailty measure, it has been shown that considering also sub-tasks of the TUG test [5] and gait speed [6] can further improve frailty assessments. In a previous work, we have developed an ultrasound-based TUG measurement device to be used autonomously by patients at risk of frailty [7]. Initial experiments for subtask detection showed promising results [8], but still left some room for improvement. This paper describes the development of advanced algorithms for subtasks detection and for step frequency estimation from TUG signals.

\footnotetext{
${ }^{1}$ Corresponding author: Andreas Ziegl, Center for Health \& Bioresources, AIT Austrian Institute of Technology, Reininghausstr. 13, 8020 Graz, Austria; E-mail: andreas.ziegl@ait.ac.at.
} 


\section{Methods}

\subsection{Recording of supervised TUG tests}

We recorded healthy volunteers performing supervised TUG tests. TUG device data were captured together with audio and video data using a camera in an Android mobile phone. Data automatically derived from the TUG recordings were compared to a ground truth, which was manually extracted from the audio and video recordings.

\subsection{Subtask classification}

We designed and compared algorithms based on three different approaches for subtask detection. For filtering and smoothing, a Gauss distribution was fit to the original TUG signal. Four transitions in between the five subtasks of the TUG test were annotated: stand up/walk forward (T1), walk forward/turn around (T2) turn around/walk back (T3) walk back/sit down (T4). Figure 1 shows an example of a test signal with the moments of the transitions marked by the vertical lines.

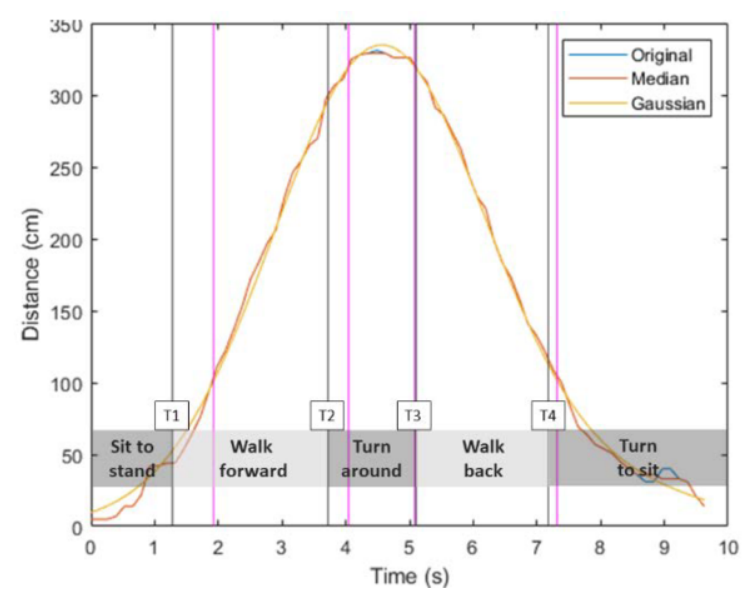

Figure 1. Example of a test signal. The curve represents the distance from the subject to the device placed in the backrest of the chair. TUG subtasks and the transitions between them according to reference are represented in gray. Transition times estimated with one of the algorithms are marked by pink vertical lines.

Approach 1 was based on our previous approach published in [8], which separated the subtasks depending on specific distances of the subject to the device. However, no reference subtask annotations were available when developing the approach in [8]. Therefore, for approach 2, we optimized the threshold distances based on the recorded videos. For approach 3, T1 and T4 were estimated before the maximum and at the minimum of the polynomial fitting of the derivatives of the signals. Table 1 presents an explanation of the different criteria established for the detection of the subtask transitions.

Table 1. Explanation of three different approaches to separate subtasks: stand up/walk forward (T1), walk forward/turn around (T2) turn around/walk back (T3) walk back/sit down (T4).

\begin{tabular}{lllll}
\hline Approach & T1 & T2 & T3 & T4 \\
\hline 1 & $1 \mathrm{~m}$ (way out) & $3.2 \mathrm{~m}$ (way out) & $3.2 \mathrm{~m}$ (way back) & $1 \mathrm{~m}$ (way back) \\
\hline 2 & $0.5 \mathrm{~m}$ (way out) & $3 \mathrm{~m}$ (way out) & $3.3 \mathrm{~m}$ (way back) & $1.2 \mathrm{~m}$ (way back) \\
\hline 3 & $\begin{array}{l}\text { Before the maximum of the } \\
\text { derivative's polynomial fitting }\end{array}$ & - & $\begin{array}{l}\text { At the minimum of the } \\
\text { polynomial fitting }\end{array}$ \\
\hline
\end{tabular}




\subsection{Step frequency}

We considered two different approaches for estimating the step frequency. In the frequency domain, after applying some processing steps to the signals, we transformed them to the frequency domain via a fast Fourier transform. We considered the highest peak in the spectrum as the estimated frequency. In the time domain, we derived the number of steps made during the test from the derivative curve of the signal. Specifically, the number of peaks higher than a certain value in the derivative signal was assessed with each of the peaks corresponding to one step. The peaks were counted for the walking period. After estimating the number of steps for each test, we calculated the step frequency dividing the number of steps in a test by the duration of the test.

\section{Results}

\subsection{Dataset}

Eight healthy volunteers (20 to 29 years, 5 female) each performed 10 tests, resulting in $80 \mathrm{TUG}$, video and audio signals. The average test duration was of $10.01 \mathrm{~s}$. The average difference between total TUG time measured by the device and respective reference values from the videos was $0.19 \pm 0.16$ seconds (mean relative error: $1.50 \%$ ).

\subsection{Analysis of the subtask classification strategies}

Table 2 summarizes the results for all the approaches. The mean time difference refers to the comparison between the real subtask transition times (extracted from the videos) and the times obtained by establishing the corresponding approach.

Table 2. Comparison of subtask times as calculated based on criteria with the ground truth derived from video recordings including correlation coefficients $\mathrm{R}$.

\begin{tabular}{lllll}
\hline Approach 1 & T1 & T2 & T3 & T4 \\
\hline R $(p-v a l u e)$ & $0.65(\mathrm{p}<0.001)$ & $0.93(\mathrm{p}<0.001)$ & $0.87(\mathrm{p}<0.001)$ & $0.97(\mathrm{p}<0.001)$ \\
Mean \pm std & $0.71 \pm 0.34 \mathrm{~s}$ & $0.28 \pm 0.24 \mathrm{~s}$ & $0.41 \pm 0.48 \mathrm{~s}$ & $0.34 \pm 0.34 \mathrm{~s}$ \\
\hline Approach 2 & T1 & T2 & T3 & T4 \\
\hline R $(p-v a l u e)$ & $0.48(\mathrm{p}<0.001)$ & $0.91(\mathrm{p}<0.001)$ & $0.86(\mathrm{p}<0.001)$ & $0.95(\mathrm{p}<0.001)$ \\
Mean \pm std & $0.25 \pm 0.27 \mathrm{~s}$ & $0.25 \pm 0.23 \mathrm{~s}$ & $0.35 \pm 0.48 \mathrm{~s}$ & $0.28 \pm 0.37 \mathrm{~s}$ \\
\hline Approach 3 & $\mathbf{T 1}$ & $\mathbf{T 2}$ & $\mathbf{T 3}$ & $\mathbf{T 4}$ \\
\hline R (p-value $)$ & $0.62(\mathrm{p}<0.001)$ & - & - & $0.97(\mathrm{p}<0.001)$ \\
Mean \pm std & $0.22 \pm 0.17 \mathrm{~s}$ & - & - & $0.27 \pm 0.22 \mathrm{~s}$ \\
\hline
\end{tabular}

\subsection{Analysis of the step frequency calculation strategies}

Estimated step frequencies were compared with the reference frequencies extracted from the videos. In the frequency domain, the difference between the measurements was considerably high and with respect to the correlation, the results were not significant. For the analysis in the time domain of the step frequency of the participants, the estimated number of steps was translated to frequency by dividing it by the total duration of the test. The mean difference and standard deviation between the real and the estimated frequencies for all subjects was $0.15 \pm 0.12 \mathrm{~Hz}(\mathrm{R}=0.45, \mathrm{p}<0.05)$. 


\section{Discussion}

Regarding the validation of the TUG device, the level of accuracy ensures that the small error would not influence the identification of the subject's frailty. Overall, it can be stated that the TUG device is also useful to detect where each of the subtasks starts and ends autonomously, avoiding the use of complex camera systems [9] or sensors [10]. For the gait speed estimation, in the frequency domain, the results cannot be considered worthwhile since a $p$-value of $p>0.05$ leads to discard this method. In the time domain, the results were significantly improved as the mean error of the estimation as well as the p-value were considerably decreased.

The newly developed algorithms have been optimized and tested based on the same test set. A prospective study is currently planned, to validate the results and to exclude potential overfitting. There may be imprecision in the results coming from the inaccuracy at the determination of the "real" parameters. If different people had determined the values for those parameters, they may not have given the exact same results.

All the investigation proceeded in this work should be extended to elderly people. It should be studied if the designed algorithms can also be applied to signals obtained from elderly people which may also have mobility limitations. All the processing steps developed could be applied to previously recorded TUG device signals for other studies. In [7] fall risk of an elderly population was assessed by discrimination between fallers and non-fallers. For this purpose, different features of the TUG test were considered. This investigation could be extended by evaluating the differences in gait speed or in the duration of specific subtasks between the two groups.

\section{References}

[1] Sukkriang N, Punsawad C. Comparison of geriatric assessment tools for frailty among community elderly. Heliyon. 2020 Sep 14;6(9):e04797.

[2] Cobo A, Villalba-Mora E, Pérez-Rodríguez R, Ferre X, Rodríguez-Mañas L. Unobtrusive Sensors for the Assessment of Older Adult's Frailty: A Scoping Review. Sensors (Basel). 2021 Apr 23;21(9):2983.

[3] Vavasour G, Giggins OM, Doyle J, Kelly D. How wearable sensors have been utilised to evaluate frailty in older adults: a systematic review. J Neuroeng Rehabil. 2021 Jul 8;18(1):112.

[4] Podsiadlo D, Richardson S. The timed "Up \& Go": a test of basic functional mobility for frail elderly persons. J Am Geriatr Soc. 1991 Feb;39(2):142-8.

[5] Ansai JH, Farche ACS, Rossi PG, de Andrade LP, Nakagawa TH, Takahashi ACM. Performance of Different Timed Up and Go Subtasks in Frailty Syndrome. J Geriatr Phys Ther. 2019 Oct/Dec;42(4):287293.

[6] Jung HW, Jang IY, Lee CK, Yu SS, Hwang JK, Jeon C, Lee YS, Lee E. Usual gait speed is associated with frailty status, institutionalization, and mortality in community-dwelling rural older adults: a longitudinal analysis of the Aging Study of Pyeongchang Rural Area. Clin Interv Aging. 2018 Jun 6;13:1079-1089.

[7] Ziegl A, Hayn D, Kastner P, Löffler K, Weidinger L, Brix B, Goswami N, Schreier G. Quantitative falls risk assessment in elderly people: results from a clinical study with distance based timed up-and-go test recordings. Physiol Meas. 2020 Dec 17;41(11):115006.

[8] Ziegl A, Kastner P, Modre-Osprian R, Schreier G. Automated Measurement and Subtask Analysis of the Timed Up-and-Go Test in the Field of Geriatrics. Annu Int Conf IEEE Eng Med Biol Soc. 2018 Jul;2018:1526-1529.

[9] Ansai JH, Farche ACS, Rossi PG, de Andrade LP, Nakagawa TH, Takahashi ACM. Performance of Different Timed Up and Go Subtasks in Frailty Syndrome. J Geriatr Phys Ther. 2019 Oct/Dec;42(4):287293. doi: 10.1519/JPT.0000000000000162. PMID: 29210935.

[10] Coulthard JT, Treen TT, Oates AR, Lanovaz JL. Evaluation of an inertial sensor system for analysis of timed-up-and-go under dual-task demands. Gait Posture. 2015 May;41(4):882-7. doi: 10.1016/j.gaitpost.2015.03.009. Epub 2015 Mar 21. PMID: 25827680. 